\title{
Strategy for Design and Building Multimedia Data Type
}

\author{
Manish Kumar \\ Asst. Professor \\ Deptt. Of Computer Science \& Engg. \\ Bhagalpur College of Engineering \\ Bhagalpur, Bihar
}

\begin{abstract}
This research work addresses a strategy for design and building of a comprehensive architecture to satisfy many information management requirements for Large and Distributed Multimedia Data. The paper proposes a unified model for designing multimedia data types which includes data representatives, content representatives. The proposed approach discusses several database architectures and user access mechanisms for data storage, efficient searching and fast retrieving of large and distributed multimedia data. The paper focused on the structure designing the data type which provides support for content based retrieval of multimedia data. The Query Language is an extension of a traditional query language which allows restrictions to be expressed on features, concepts of object of multimedia data
\end{abstract}

\section{Keywords}

Multimedia

\section{MULTIMEDIA DATABASES}

There is tremendous growth in multimedia data in application domains such as medicine, engineering, biology and numerous others. New technologies such as computer tomography or digital photography produce increasingly large volumes of data. At the same time, decreasing storage prices allow archiving large amounts of multimedia data at relatively low cost. For database technology, large multimedia databases require efficient and effective strategies for accessing and processing of multimedia data. Examples of typical multimedia content are music, medical magnetic resonance images, and shape and color images.

\subsection{Multimedia Object Types}

Objects can be classified in terms of their presentation and application lifetimes.

Persistent object: A persistent object is one that can exist for the duration of the application in a persistent form.

Non persistent: A "non-persistent" object is created dynamically and discarded when obsolete.

For presentation a "transient" object is denied as an object that is presented for a short duration without manipulation. The display of a series of audio or video frames represents transient presentation of objects, whether created dynamically or retrieved from a database. Objects are "static" during presentation if they exist for an extended period for their possible manipulation. A still image is an example of a static object. A Multimedia database (MMDB) differs from a conventional database in that its content may consist of types that are not commonly found in conventional databases. Multimedia now-a-days, is perhaps the most diverse and rich culture around the globe. The diversity of this aspect of multimedia leads to one dilemma and that is a variety of media formats. Currently, richly used Media Formats such as, Video (MPEG, MOV, WMV etc.), Audio (MP3, MIDI etc.), Image (JPEG, GIF etc.) and Documents (PPT, PDF, TXT etc.)i serve as a source of information and archival and thus the fact arises that such a large collection of media should be organized and properly indexed for search and retrieval in a standard way, keeping aside the with the availability of tremendous amount of digitized multimedia content (from the WWW to the CD-ROMs and video disks) and the increasing affordability of disk space in computers, people and organizations can now consider having multimedia, without involving physical effort of a person. The main focus is on two of the ORDMS market leaders: IBM's DB2 and Oracle. The salient facilities of DB2 and Oracle in handling object types are analyzed, considering their advantages and disadvantages.ii The research area has chosen to focus on the following areas:

- User defined types (UDTs)

- Processing and manipulation of large object types (LOB) and their extensions

- Creating, inserting, and updating objects

- Inheritance

- Polymorphism

GBIS (the Graphical Benchmark Information Service)iii iv, DBbrowser $\mathrm{v}$ vi and EASIA (Extensible Architecture for Scientific Data Archives)vii viii, each of which has provided the basic ideas for MMDB in the fast evolving Web environment.

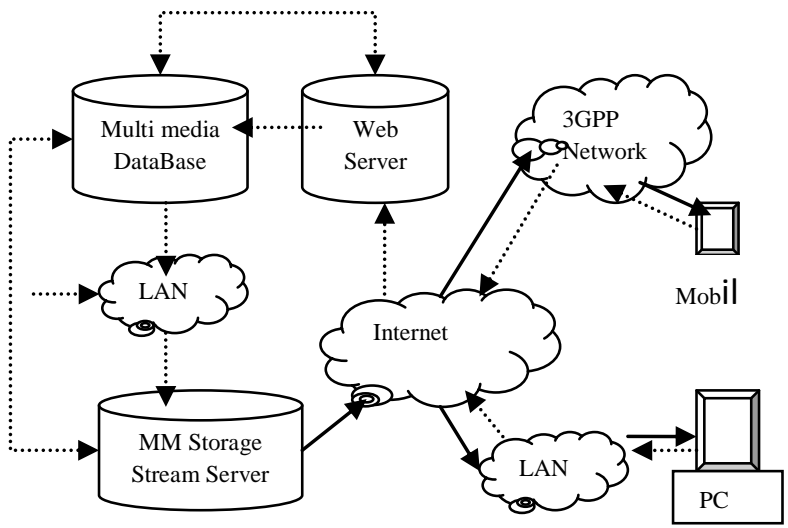


Fig 1: Components and information flow of a typical distributed Multimedia System and databas

MMDBMS manages the following relations:

- Attribute Relation: supports different presentation (audio, video, image) of one object

- Component Relation : includes all parts belonging to one data object

- Substitution Relation: defines different kinds of presentation of the same information, e.g. equation as tables, graphs, animation

- Synchronization Relation: describes temporal relations between data units, e.g. lip synchronization of audio and video.

- Real-time data transfer: DBMS must perform read and write operations of continuous data in real-time the data transfer of continuous data has a higher priority than other database management actions. Primitives of multimedia operating systems should be used to support the real time transfer of continuous data.

- Long transactions: The transfer of large amounts of data will take a long time and must be done in a reliable fashion

\subsection{Requirements of a MDBMS}

Multimedia objects are different from traditional text or numerical documents in the way that multimedia objects usually require a large amount of memory and disk storage. Also, the operations applied to multimedia objects are different (e.g., displaying a picture or playing a video clip, which is different from displaying a text paragraph). A multimedia database management system should be able to provide the following basic functions:

- Handles image, voice, and other multimedia data types

- Handles a large number of multimedia objects

- Provides a high-performance and cost-effective storage management scheme

\section{COMBINING THE BENEFITS OF OBJECT-ORIENTED AND RELATIONAL TECHNOLOGY}

OODBs have the potential to reduce the difficulty of designing large complex databases and applications. Inheritance and encapsulation make database design and application program reuse possible. These features include a full non-procedural query language, views, and dynamic schema changes and parameterized performance tuning. Added to this, the robustness, scalability and fault tolerance of OODBs does not meet that of more mature RDBs. This combination makes it possible to support dynamic schema changes, SQL, triggers, constraints, automatic query optimization, and views as a unit of authorization.

Both the GBIS and DBbrowse prototypes were implemented using CGI technology largely because this was the emerging mechanism for implementing interactive HTML pages at that time. GBIS used UNIX shell scripting for implementing the CGI programs, while DBbrowse was written using PERL CGI programs. DBbrowse performed a new database connection for each query, even for subsequent queries submitted by the same user. This leads to performance degradation.

The EAMM prototype does not use the CGI mechanism. Instead EAMM uses Java Servlets with connection pooling to remove the process per request overhead associated with CGI, to maintain state between requests and to maintain database connections for a complete client session., Java was used server-side. The portability of Java code and the extensive APIs (or class libraries, for example JBDC) provided great benefits. Java Servlets were used to implement server-side logic in the EAMM prototype. A Java Servlet ix is a dynamically loaded Java class that extends the capabilities of a Web server. Once loaded a Servlet remains in memory, and is handled by separate threads within the Web server process. This provides a much better performance than CGI, which creates a new process for each request. Orfali and Harkey $\mathrm{x}$ found that Servlets performed over an order of magnitude better than CGI in a 'ping' benchmark.

Servlets are written in Java and, unlike the previously discussed non-standard CGI workarounds, are supported by all major Web servers. Servlets were unavailable when the GBIS and DBbrowse prototypes were implemented. However, Servlet technology was used to implement EAMM.

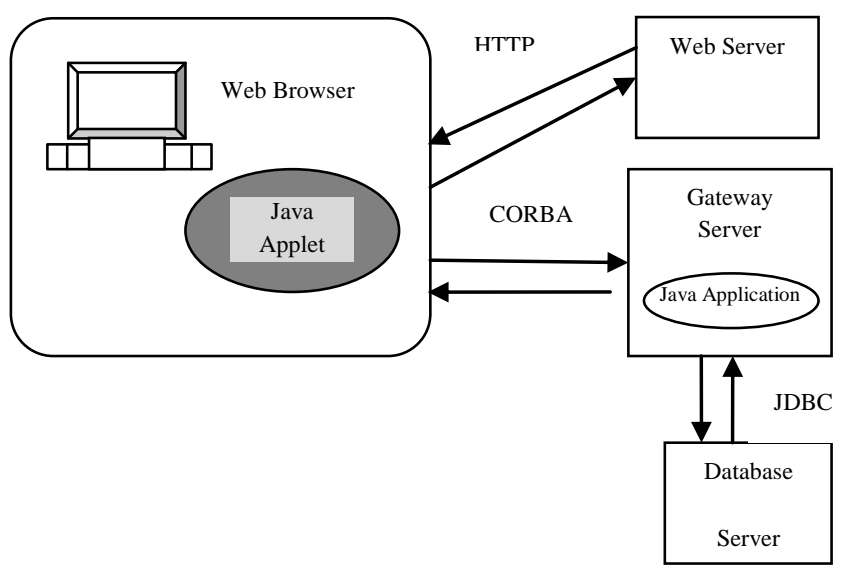

Fig 2: A 3-tier architecture using a Java Applet, CORBA and JDBC

\section{BASIC APPROACHES FOR DATA RETRIEVAL}

Conventional Database System: This is the widely-used approach to manage and search for structured data. All data in a database system must conform to some predefined structures and constraints.

Information Retrieval (IR) System : IR system is mainly used to search large text collections, in which the content of the (text) data is described by an indexer using keywords or a textual abstract, and keywords or natural language is used to express query demands.

Content Based Retrieval (CBR) system: This approach is being used to retrieve desired multimedia objects from a large collection on the basis of features (such as color, texture and shape, etc.) that can be automatically extracted from the objects themselves. The most common characteristics used for content based retrieval are: texture, color and shape xi xii xiii xiv. This method presents an application that includes a dedicated MMDBMS server based on the SQL3 standard, which is less expensive than a commercial database server. 


\subsection{Image Representation in the Database Systems}

The logical image representation in image databases systems is based on different image data models. An image object is either an entire image or some other meaningful portion (of an image. The logical image description includes: Meta, semantic, color, texture, shape, and spatial attributes.

The mid 90s saw a first wave of commercial, implemented from-the-scratch, and full-fledged MMDBMS. Some of them were MediaDB, now MediaWayxv, JASMINE xvi, and ITASCA that is the commercial successor of ORION xvii.

\section{CONCLUSIONS}

The main focuse on the design of a MDBMS that can efficiently organize, store, manage, and retrieve multimedia information from the underlying multimedia database, as well as to implement a multimedia database, we can use relational database model where we can predefine the relation among multimedia objects using OODB concepts.

\section{REFERENCES}

[1] Papiani, M., Hey, A.J.G. and Hockney, R.W. The Graphical Benchmark Information Service. Scientific Programming, Vol. 4(4), 1995, 219-227.

[2] Alexander P. Pons, Hassan Aljifri , Handling Unstructured Data Type in DB2 and Oracle, Communications of the International Information Management Association, Volume 3 Issue 2

[3] Papiani, M., Hey, A.J.G. and Hockney, R.W. The Graphical Benchmark Information Service. Scientific Programming, Vol. 4(4), 1995, 219-227.

[4] Hockney, R., W. The Science of Computer Benchmarking, Philadelphia, USA, SIAM, 1996, 104115. ISBN 0-89871-363-3.

[5] Papiani, M., Dunlop, A., N. and Hey, A., J., G. Automatic Web Interfaces and Browsing for ObjectRelational Databases. Advances in Databases: Proceedings of the 15th British National Conference on Databases, BNCOD15, London, 7-9th July, 1997, 131-2.
[6] Dunlop, A., N., Papiani, M. and Hey. A.J.G. Providing Access to a Multimedia Archive Using the World-Wide Web and an Object-Relational Database Management System. IEE Computing \& Control Engineering Journal, Vol. 7(5), October 1996, 221-6.

[7] Papiani, M., Wason, J., L. and Nicole, D., A. An Architecture for Management of Large, Distributed, Scientific Data Using SQL/MED and XML. Proceedings of the 7th International Conference on Extending Database Technology (EDBT), Konstanz, Germany, March, 2000, 447-61.

[8] Papiani, M., Wason, J., L., Dunlop, A., N. and Nicole, D., A. A Distributed Scientific Data Archive Using the Web, XML and SQL/MED. ACM SIGMOD Record, Vol. 28(3), September, 1999, 56-62.

[9] Davidson, J., D., and Ahmed, S. Java Servlet API Specification, Version 2.1a, November, 1988 http://java.sun.com/products/servlet/index.html

[10] Orfali, R. and Harkey, D. Client/Server Programming with JAVA and CORBA, 2nd edn, John Wiley \& Sons, Inc., 1998, 1022pp.

[11] Del Bimbo, A. (2001): Visual Information Retrieval, Morgan Kaufmann Publishers. San Francisco USA

[12] Faloutsos, C. (2005): Searching Multimedia Databases by Content. Springer Gevers, T., Smeulders, W.M. (1999): Color-based object recognition. Pattern Recognition. 32, 453- 464

[13] Gevers, T. (2001). Color in Image Search Engines. Principles of Visual Information Retrieval. SpringerVerlag, London

[14] Gevers, T., Smeulders, W.M. (1999): Color-based object recognition. Pattern Recognition. 32, 453- 464

[15] B. Phillips, Mediaway presses access to multimedia database. PC Week, 13(7), 1996, 39-40.

[16] D. Woelk, W. Kim, \& W. Luther, An object-oriented approach to multimedia databases. ACM SIGMOD, Record, 15(2), 1986, 311-325

[17] Setrag Khoshafian, Surapol Dasananda, \& Norayr Minaasian, The Jasmine Object Database: Multimedia Applications for the Web (Morgan Kaufmann Publishers, 1998). 